\title{
A simple approach toward quantitative phase field simulation for dilute-alloy solidification
}

\author{
C.J. Shih, M.H. Lee, C.W. Lan* \\ Department of Chemical Engineering, National Taiwan University, Taipei 10617, Taiwan, ROC
}

Received 19 May 2004; accepted 9 May 2005

Available online 11 July 2005

Communicated by D.T.J. Hurle

\begin{abstract}
We present a simple approach that could help quantitative phase field simulation for dilute-alloy solidification. The proposed approach mends the solute trapping problem by using a simple interface model (SIM), which requires only one free parameter. To test the feasibility of this model, a free dendritic growth from a small nucleus is simulated, and a good agreement with the anti-trapping current (ATC) model [Karma, Phys. Rev. Lett. 87 (2001) 115701] is obtained. By further studying the solute trapping effect during directional solidification, we find that the results give, with this model, a good thermodynamic consistence without solute trapping over two-order increment of the solidification speed and the interface thickness. Good agreement with the classical theory is obtained as well.
\end{abstract}

(C) 2005 Elsevier B.V. All rights reserved.

PACS: 68.70. +w; 81.30.Fb

Keywords: A1. Adaptive phase field simulations; A1. Interface model; A1. Solidification; A1. Solute trapping

\section{Introduction}

The phase field method has emerged as a powerful tool to simulate microstructure evolution in solidification [1-4]. However, limited by computation and inherent numerical nature, the phase field model has encountered many difficulties. One of the most important limitations in the phase field

\footnotetext{
*Corresponding author. Tel./fax: 886223633917.

E-mail address: cwlan@ntu.edu.tw (C.W. Lan).
}

simulation is the interface thickness $\delta$. The choice of $\delta$ needs to be small enough so that the sharpinterface limit can be achieved. However, such a value, which is usually in the order of the microscopic capillary length $d_{0}$, is several orders smaller than the scale of microstructures. On the other hand, the length scale of solutal and thermal boundary layers could be several orders larger than that of microstructures. As a result, such a sharp interface requirement is too stringent for a realistic simulation, even with today's supercomputers. 
Significant progress has been made by Karma and Rappel [3] for the thin-interface limit that $\delta$ can be chosen in the same order of the scale of microstructure. Through their asymptotic analysis, not only can the kinetic behavior be predicted well comparing with the theoretical solutions, but also the corrections to the transport equations show good agreement with the analytical ones. With such an implementation, efficient dendritic growth simulations have been reported even at low supercoolings [5,6]. Recently, Amberg [4] has further successfully reduced the problem to the interface only, which can be solved analytically, and this allows a thicker interface to be used.

For the solidification of alloys, because the solute partition occurs during phase transformation, the thick interface often generates side effects that destroy the thermodynamic relations at the interface. These $\delta$-dependent non-equilibrium effects can be classified into three parts [7]: (1) interface stretching; (2) surface diffusion along the arc length of the interface; and (3) discontinuity of chemical potential. To achieve a quantitative simulation, all of these three terms need to be eliminated, so that the thermodynamic consistence and mass conservation can be satisfied locally. Unfortunately, the thin-interface analysis has showed that it is only possible to correct any two of the three effects by selecting adequate weight/ interpolation functions. Eliminating all the three terms at the same time is not possible unless some modifications are considered. The introduction of the anti-trapping current (ATC) to the diffusion equation proposed by Karma [7] is a good approach to the solution. The idea of ATC tries to import an artificial solute current to the diffusive interface, so that the solute trapping effect resulting from the discontinuity of chemical potential jump can be compensated. This model also gives some freedom in selecting appropriated interpolation and weighting functions to eliminate the other effects. As a result, a quantitative simulation of alloy solidification with a reasonable interface thickness becomes possible [7,8].

In this paper, we propose a simple interface model (SIM) that can also eliminate the nonequilibrium effects allowing quantitative alloy phase field simulations using a reasonable interface thickness. The scheme, for the same purpose as ATC, tries to suppress the solute trapping effect at the interface by considering the effects of the interface thickness and the solidification speed. For comparison purposes, we first repeat the simulation of the isothermal alloy dendritic growth in [9], and perform a one-to-one benchmark comparison. With the success, the directional solidification of a succinonitrile $(\mathrm{SCN})$ /acetone alloy is further simulated quantitatively. By using the present model to the phase field simulation, the interface thickness and solidification speed can be relaxed up to two orders, as compared with the ones using the traditional WBM model [2].

\section{Simple interface model (SIM)}

As a starting point, we derive the phase field equation from the local geometrical description following Beckermann et al. [10]. For a phase field variable $\phi(x, y, t)$ that changes rapidly from 0 (solid) to 1 (liquid) at the interface, we can simply use a hyperbolic function for the variation of $\phi$ in the normal coordinate $n$ :

$\phi=\frac{1}{2}\left[1+\tanh \left(\frac{n}{2 \delta}\right)\right]$.

Then, the unit normal vector $\mathbf{n}$, the local curvature $\kappa$, and the interface speed $V_{n}$ can be related to the phase field variable by $\mathbf{n}=\nabla \phi /|\nabla \phi|$, $\kappa=\nabla \cdot \mathbf{n}=1 /|\nabla \phi|\left(\nabla^{2} \phi-\nabla \phi \nabla|\nabla \phi| /|\nabla \phi|\right), \quad$ and $V_{n}=(-1 /|\nabla \phi|)(\partial \phi / \partial t)$. By substituting these into the generalized Gibbs-Thompson (G-T) equation (for a dilute alloy of species $A$ having an isotropic surface energy $\alpha_{A}$ ), i.e.,

$T_{i}=T_{m A}-\Gamma \kappa+m c_{L}-\frac{V_{n}}{\mu_{A}}$,

we can obtain a phase field equation as the following:

$$
\begin{aligned}
\frac{\partial \phi}{\partial t}= & \Gamma \mu_{A}\left[\nabla^{2} \phi-\frac{\phi(1-\phi)(1-2 \phi)}{\delta^{2}}\right] \\
& -\mu_{A}\left(T_{m}^{A}-T+m c_{\mathrm{L}}\right)\left[\frac{\phi(1-\phi)}{\delta}\right],
\end{aligned}
$$

where $\Gamma=\sigma_{A} T_{m}^{A} / L_{A}$ is the G-T coefficient. $L_{A}$ is the heat of fusion, $\mu_{A}$ the kinetic coefficient, and $m$ 
the slope of liquidus temperature line $\left(T_{i}\right) ; T_{m A}$ is the melting point of pure $A$ and $c_{\mathrm{L}}$ the concentration of the solute $\mathrm{B}$ at the liquid phase side.

By further considering the crystalline anisotropy, Eq. (3) in two dimensions can be rearranged to such that $p^{\prime}(\phi)=30 g(\phi)$, where $g(\phi)$ is a doublewell function; $g(\phi)=\phi^{2}(1-\phi)^{2}$. This set of selections used is promising in eliminating the stretching and surface diffusion effect under the thin-interface analysis scheme [7] (two of three

$$
\begin{aligned}
& \eta^{2} \frac{\partial \phi}{\partial t} \\
& \quad=\frac{\sigma_{A} T_{m}^{A} \mu_{A}}{L_{A}}\left[\nabla \cdot\left(\eta^{2} \nabla \phi\right)-\frac{\partial}{\partial x}\left(\eta \eta^{\prime} \frac{\partial \phi}{\partial y}\right)+\frac{\partial}{\partial y}\left(\eta \eta^{\prime} \frac{\partial \phi}{\partial x}\right)-\frac{\phi(1-\phi)(1-2 \phi)}{\delta^{2}}\right]-\mu_{A}\left(T_{m}^{A}-T+m c_{\mathrm{L}}\right)\left[\frac{\phi(1-\phi)}{\delta}\right],
\end{aligned}
$$

where the anisotropic function $\eta$ is defined for the four-fold symmetry [2], i.e., $\eta=1+\gamma \cos 4 \beta$, where $\gamma$ is the intensity of the anisotropy and $\beta=$ $\tan ^{-1}[(\partial \phi / \partial y) /(\partial \phi / \partial x)]$ determining the growth orientation of the cells.

This model can be viewed as an intermediate between the standard phase field formulation, which is derived from the non-equilibrium thermodynamics, and the classical sharp interface model (using the G-T equation at the interface). To formulate the partition of solute in the diffusive interface, we introduce an artificial segregation coefficient $k_{a}$, such that $c_{\mathrm{S}} / c_{\mathrm{L}}=k_{a}$ for the liquid $\left(c_{\mathrm{L}}\right)$ and solid $\left(c_{\mathrm{S}}\right)$ concentrations related to the average concentration $c$ of $\mathrm{B}$ as

$c_{\mathrm{L}}=\frac{c}{p(\phi)+k_{a}(1-p(\phi))}$

and

$c_{\mathrm{S}}=\frac{k_{a} c}{p(\phi)+k_{a}(1-p(\phi))}$,

respectively. Here, $p(\phi)$ is an interpolation function to describe solid-melt mixture properties. The concentration equation can also be derived from the conservation of solute as

$\frac{\partial c}{\partial t}=\nabla \cdot\left[(1-p(\phi)) D_{\mathrm{S}} \nabla c_{\mathrm{S}}+p(\phi) D_{\mathrm{L}} \nabla c_{\mathrm{L}}\right]$.

To focus on the quantitative simulation during solidification, the weighting function $\mathrm{p}(\phi)$ is adopted similar to that used the WBM model [2], non-equilibrium effects). Besides, if we transform the last term of Eq. (3) into $5 \phi^{2}(1-\phi)^{2} / \delta$, and let $p(\phi)=\phi, D_{\mathrm{s}}=0$ (one-sided model), the present phase-field model (Eqs. (3)-(5)) is identical to the Karma's model [7], except the ATC term in the species transport equation used in his model.

Now, the only problem left over in the above model is how to suppress solute trapping effect by eliminating the discontinuity of chemical potential jump. In a typical phase field simulation, because the interface is finite and diffusive, $k_{a}$ is affected by the interface thickness and the solidification speed, so that it is not the same as the equilibrium segregation coefficient $k$ unless the interface thickness is near the sharp interface limit. In other words, with a thick interface the calculated partition, or the effective segregation coefficient $k_{\text {eff }}=c_{\mathrm{S}} / c_{\mathrm{L}}$, is greater than the equilibrium $k$ if $k<1$ during solidification. Fortunately, if one can find an interface model such that $k_{\text {eff }}\left(\delta, V_{n}, k_{a}\right)=k$, a quantitative phase field simulation at any $\delta$ and $V_{n}$ is possible without the apparent solute trapping. This is the key idea for the present approach.

Therefore, we have taken a SIM that correlates first the effective segregation coefficient $k_{\text {eff }}$ with the interface diffusion speed $V_{\mathrm{D}}$ and the thickness $\delta$ as:

$V_{\mathrm{D}}=a \frac{D_{\mathrm{L}} \ln \left(1 / k_{a}\right)}{\delta\left(1-k_{a}\right)}$, 
where $a$ is a free parameter. This formulation is similar to the one proposed by Aziz [12] and Ahmad et al. [13], but the free parameter $a$ needs to be determined. In addition, we can further correlate $V_{\mathrm{D}}$ with $V_{n}$ similar to that in Refs. $[12,13]$

$k_{\mathrm{eff}}=\left(k_{a}+V_{n} / V_{\mathrm{D}}\right) /\left(1+V_{n} / V_{\mathrm{D}}\right)$.

By combining Eq. (6) and (7), we can come out a new interface model, i.e., $k_{\text {eff }}\left(\delta, V_{n}, k_{a}\right)=c_{\mathrm{S}} / c_{\mathrm{L}}=$ $k ; c_{\mathrm{S}}$ and $c_{\mathrm{L}}$ are the extracted solid and liquid concentrations, respectively, from the two sides of the interface. Since $k_{a}$ is determined by $a$ implicitly, one can rewrite the model as $k_{\text {eff }}\left(\delta, V_{n}, a\right)=k$ such that the best-fit parameter $a$ can be determined by several one-dimensional simulations under various $\delta$ and $V_{n}$ easily. In other words, if we have the model, given the normal velocity and the interface thickness, we can calculate $k_{a}$, which is required in the phase field equations having $c_{\mathrm{S}} / c_{\mathrm{L}}=k_{\mathrm{eff}}=k$. Unlike the classical phase field models $[2,7,10]$, the $k_{a}$ in this model is an adaptive parameter in Eq. (5), which depends on the interface thickness and solidification speed (Eqs. (6) and (7)), to restore $k_{\text {eff }}$ as a desired value automatically. Moreover, if the interface thickness $\delta$ is much less than the tip radius, this local model is approximately onedimensional and is independent of the convection outside of the interface.

As just mentioned, the goal of the thin-interface analysis is to compensate the local mass unbalance for the finite and diffusive interface. The key of ATC is to correct the discontinuity of chemical potential, and as a consequence of relaxing more freedom to choose the weight/interpolation function to correct the other two effects. However, in the present SIM model, it is straight forward and engineer-intuitive, since we change the equilibrium partition coefficient $k$ to compensate the mass balance directly. Moreover, in SIM, $k_{a}$ is determined according to magnitude of concentration, normal velocity and interface thickness, which has the same spirit of ATC. The main difference is our free parameter $a$ is determined empirically, but the ATC coefficient [7] is determined by matching the asymptotic analysis.

Finally, for the selection of the kinetic coefficient, the thin-interface limit [3] is considered as the following:

$\frac{1}{\mu_{k}}=\left[5 a_{2} / 4\right]\left[\delta / D_{\mathrm{L}}\right]\left[\left(D_{\mathrm{L}} L / \alpha C_{p}\right)-m(1-k) c\right]$,

where $a_{2}=0.6267$ [3], $\alpha$ the thermal diffusivity, and $C_{p}$ the heat capacity. For simplicity, the constant $\mu_{k}$ under the uniform concentration field $c=c_{0} / k$ is used here, where $c_{0}$ is the bulk concentration.

\section{Results and discussion}

\subsection{Free dendritic growth of a binary alloy}

To benchmark our scheme with the ATC model [7], we consider the isothermal dendritic growth of an alloy that has been simulated quantitatively by Ramirez et al. [9]. For comparison purpose, the last term of Eq. (3) is changed into $5 \phi^{2}(1-\phi)^{2} / \delta$, and $p(\phi)=\phi$, and $D_{\mathrm{s}}=0$ (one-sided model). Hence, besides the ATC term, our model is the same as the one used by Ramirez et al. if we simply rearrange the variables used in Ref. [9] by $W=\sqrt{2} \delta, \tau=2 \delta^{2} / \mu_{k} \Gamma$, and the coupling constant $\lambda=5 \delta / 4 \Gamma$. Then, the value of free parameter $a$ in Eq. (6) is determined to be 1.27 for the model proposed here. We perform simulation using the adaptive finite volume method [6] to optimize the efficiency and accuracy. The minimum grid size $\Delta x_{\min }$ is controlled by maintaining $\Delta x_{\min } / W \leq 0.4$. Also, the kinetic coefficient is set to be the same as what thin interface limit suggests under the constant concentration; other parameters used [9] are adopted as well.

The solute profile in the solid is shown in Fig. 1. As shown, both ATC and SIM models suppress the solute-trapping effect successfully as compared with the standard one. The solidus concentration is also in a good agreement with the G-T solution, i.e., $c_{\mathrm{s}}=k\left[\left(1-(1-k) d_{0} / \rho\right]\right.$, where $\rho$ is the steadystate tip radius obtained from the simulations at $W / d_{0}=1.805$ and $d_{0}$ the thermal capillary length [9]. The time evolution of the tip speed and radius under various interface thickness are sketched in Figs. 2 and 3, respectively. For the ones having the same interface thickness $\left(W / d_{0}=3.61\right)$, the proposed model shows significant improvement 


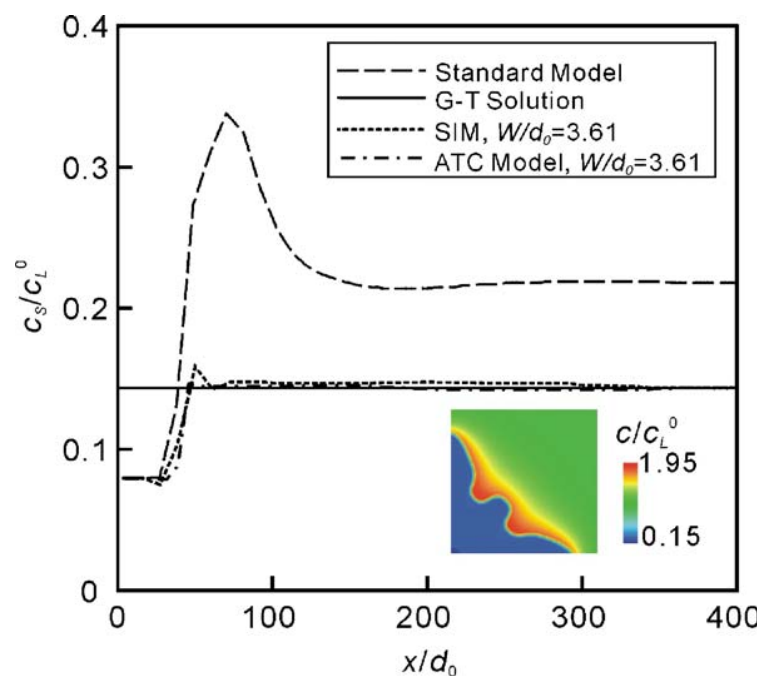

Fig. 1. Comparison of simulated solidus concentration profiles by using different phase field models.

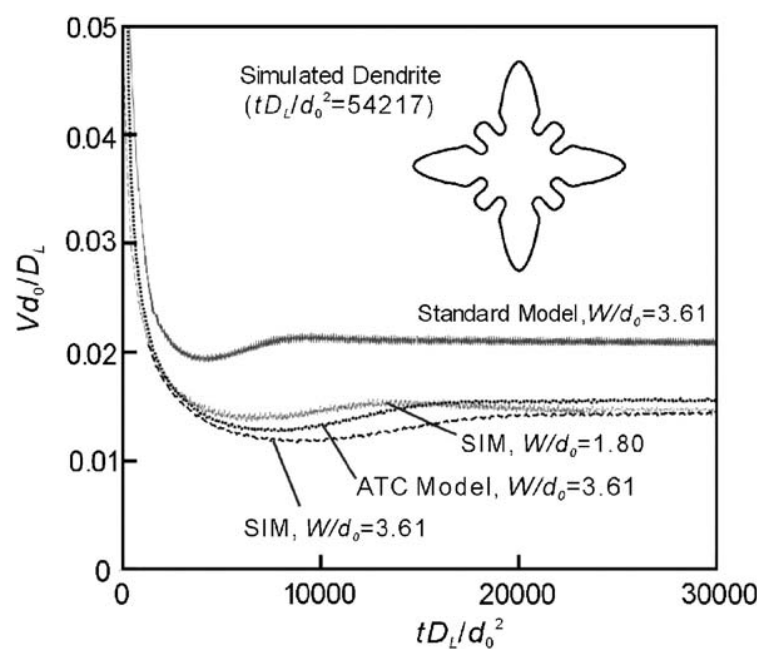

Fig. 2. Comparison of calculated tip speeds by using different phase field model and various interface thicknesses.

comparing with the 'standard' model [7], as well as a good agreement with the ATC model. As compared with the ATC model, we find that the deviations of the calculated steady-state tip speed and tip radius are less than $5 \%$.

The convergence of the steady tip speed with the interface thickness is plotted in Fig. 4. Our

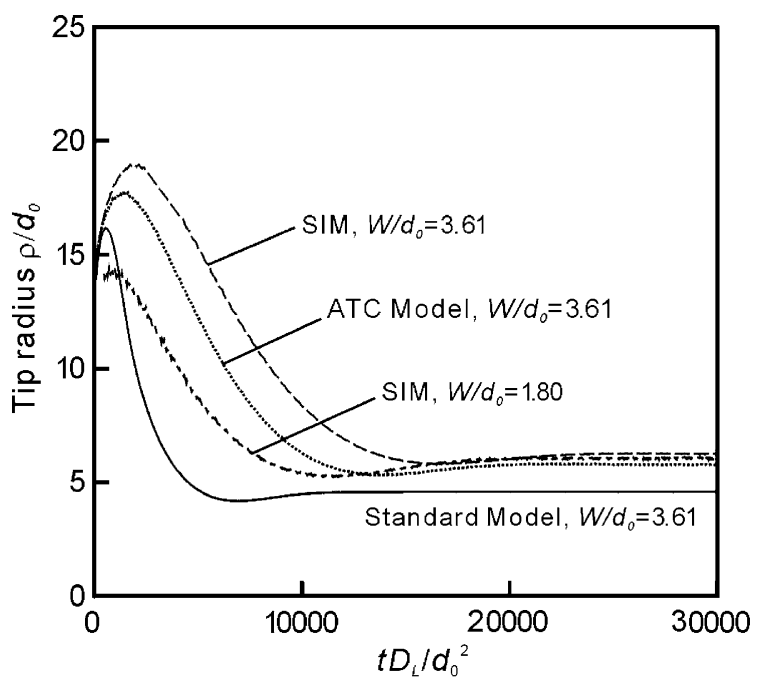

Fig. 3. Comparison of calculated tip radius by using different phase field model and various interface thicknesses.

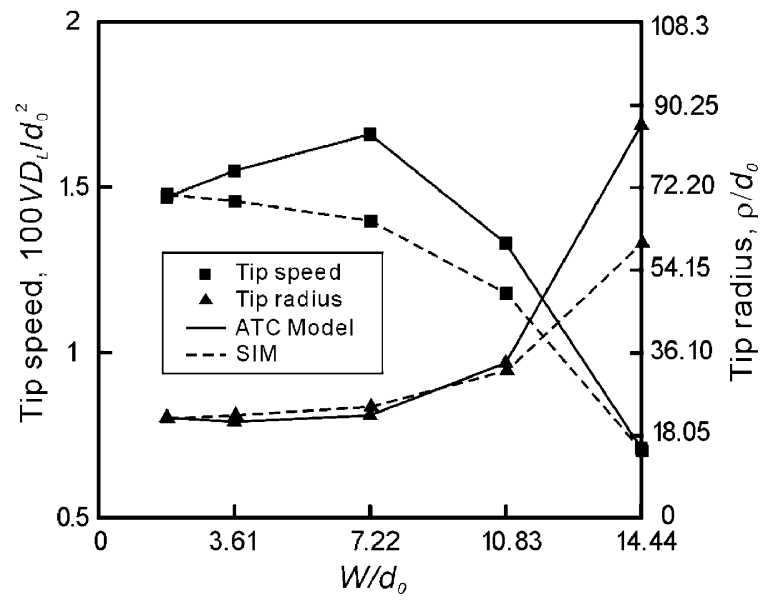

Fig. 4. Convergence of tip speed and radius with the interface thickness by using the ATC model and our present SIM model.

convergence study shows nearly the same as that in Ref. [9] at the thinnest interface thickness. Theoretically, both SIM and ATC schemes should converge to the same thin interface limit, because $k_{a}=k$ and ATC term vanishes as the interface thickness approaches to zero. Therefore, the same convergence shown in Fig. 4 does not surprise us. 
Also, we only plot the SIM results for $W / d_{0}=1.805$, because both the results match nicely and is difficult to tell the difference. On the other hand, for a thick interface, our model indeed eliminates the effect of solute trapping, and the performance is comparable to the ATC model. Interestingly, for a much thicker interface thickness $\left(W / d_{0}>10.83\right)$, the tip speed becomes slower and slower and it is hard to reach a steady state even after a long growth time, which shows similar trend as the ones simulated by Ramirez et al. [9]. This anomalous deviation for $W / d_{0}>10.83$ is due to the interface thickness being too close to the local tip radius. Accordingly, it is difficult to get an accurate tip morphology with this thick interface. In other words, just like other quantitative phase field models $[3,4,7]$, the restriction of our model is that the interface thickness should be much smaller than the local tip radius.

\subsection{Directional solidification of a dilute $S C N /$ acetone alloy}

Once we have built the confidence in the present model, we can further apply the model to the directional solidification of a thin-film alloy. By considering a directional solidification system in a moving frame with a stable speed $V$, the timederivative terms in Eqs. (4)-(5) can be rewritten as $\partial \phi / \partial t-V(\partial \phi / \partial y)$ and $\partial c / \partial t-V(\partial c / \partial y)$, respectively. To examine the present model, we take the directional solidification of SCN containing $0.05 \mathrm{~mol} \%$ acetone as an example $(k=0.1$, $m=-222 \mathrm{~K} / \mathrm{mol}$ frac.), while the physical properties are taken from SCN directly. A linear temperature profile having a gradient $G$ of $100 \mathrm{~K} / \mathrm{cm}$ is used for simulation. First, we examine the situation having a planar growth front at a low solidification speed $V=10 \mu \mathrm{m} / \mathrm{s}$ using a thick interface thickness $\delta=5 \mu \mathrm{m}$. The artificial segregation coefficient $k_{a}$ is set to be the equilibrium one $\left(k_{a}=k=0.1\right)$, i.e., using a standard phase field model. During simulation, the interface temperature, acetone concentration $\left(c_{\mathrm{L}}\right.$ and $\left.c_{\mathrm{S}}\right)$, and effective segregation coefficient $\left(k_{\text {eff }}=c_{\mathrm{L}} / c_{\mathrm{S}}\right)$ are recorded. As shown by the square symbols (standard phase field model) in Fig. 5, the calculated results deviate from the phase diagram

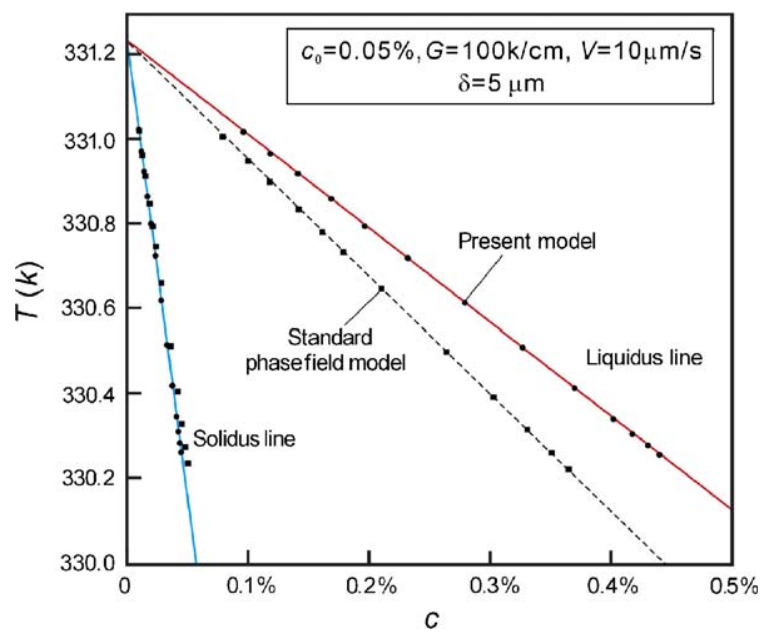

Fig. 5. Comparison of the simulated solid and liquid concentration with the $\mathrm{SCN} /$ acetone phase diagram at the planar front for $V=10 \mathrm{~m} / \mathrm{s}$ and $c_{0}=0.05 \mathrm{~mol} \%$.

significantly, especially on the liquidus line. The acetone partition ratio, or the effective segregation coefficient, is also larger $\left(k_{\text {eff }}=0.125\right)$ than the equilibrium one $(k=0.1)$. Again, this deviation is due to the solute trapping effect caused by the finite interface thickness. The solute trapping or $k_{\text {eff }}$ increases with the interface thickness (Fig. 6a) and the solidification speed (Fig. 6b) quickly. Due to this limitation, the quantitative simulation of a solidification problem having a wide range of the solidification speed in a large domain becomes extremely difficult. By using the SIM model, through several one-dimensional simulations, which are very easy to do, we have got a best-fit value of $a=0.56$ in this case. By using the SIM with $a=0.56$, as shown by the dot symbols in Fig. 5, the solute trapping effect is almost removed. The effect of solute trapping can be totally compensated, while the calculate segregation coefficient $k_{\text {eff }}$ is independent of interface thickness. Furthermore, this model can be extended to a much wider range of the pulling speed and interface thickness, as plotted in Figs. 6a and $6 \mathrm{~b}$, respectively. As shown, both the solidification speed and the interface thickness are significantly increased about two orders in the magnitude without the introduction of solute trapping. 

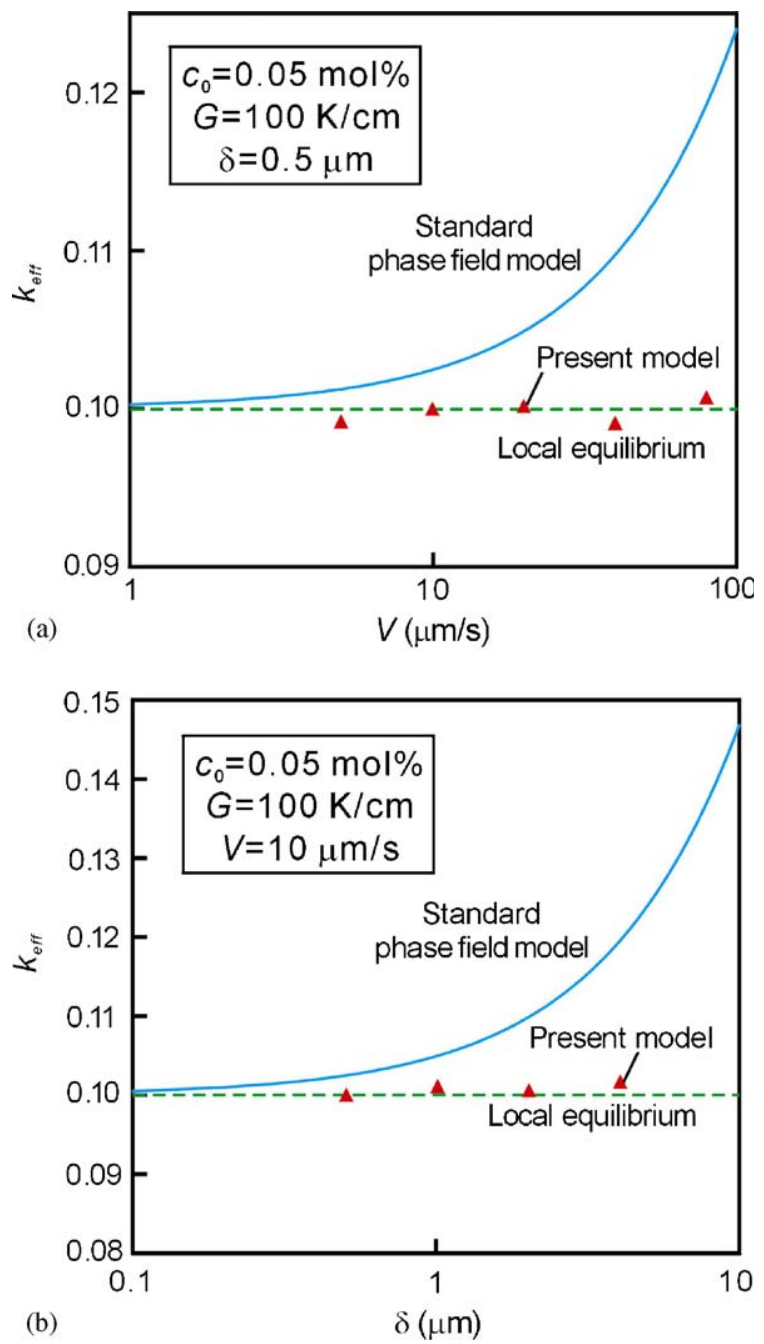

Fig. 6. Comparison of simulated $k_{\text {eff }}$ as a function of (a) pulling speed and (b) interface thickness by using the proposed model with the standard model.

Although the solute trapping could be reduced even with a large interface thickness, which saves significant computation time, the accuracy on the cell morphology could be degraded rapidly as we discussed in Fig. 4. Fig. 7 shows the calculated solute and phase field contours at various interface thickness $\left(c_{0}=0.05 \mathrm{~mol} \%, G=100 \mathrm{~K} / \mathrm{cm}\right.$, and $V=16 \mu \mathrm{m} / \mathrm{s})$. At first, we use a thick $\delta(1 \mu \mathrm{m})$ to simulate morphological evolution from a planar interface, while the number of control volumes at the steady state is about 30000 . However, to obtain clear structure of grooves, the interface thickness needs to be decreased stepwise down to $0.5 \mu \mathrm{m}$, and the number of control volumes is increased to 100000 . As shown, the solute and phase-field profiles above the dashed line are almost unchanged for different $\delta$ 's, while the groove structure is highly thickness dependent. Furthermore, we notice that the phase-field is stretched seriously if the local radius is comparable to the interface thickness, which is also observed in the simulation of Widmanstätten ferrite [14]. Interestingly, once is thin enough to describe local interface, the phase-field shrinks quickly, and the reminder becomes pinched-off drops. Further decreasing the interface thickness does not change the results.

It is well known that in a directional solidification experiment of an alloy, the planar interface could become unstable and wrinkled as the pulling speed is greater than a critical value $V_{c}$, as predicted analytically by the classic Mullins-Sekerka (MS) theory [11]. This morphological instability occurs because the constitutional supercooling overcomes the stabilizing effects of the thermal gradient and interfacial tension. As observed in the experiments, once the instability starts, shallow cellular structures grow initially followed by deep cells and then dendrites with the increasing speed. However, its simulation has not yet been simulated quantitatively by a phase field model so far. The use of a large interface thickness makes the long-time scale simulation (in the order of $D_{\mathrm{L}} / k V^{2}$ [15], up to several minutes here) possible for the morphological instability at high concentration, where the solidification speed is small. The simulated critical $(G / V)_{\mathrm{c}}$ for the instability at various acetone concentrations are further summarized in Fig. 8. As shown, good agreement with MS theory is obtained; the results are obtained by reducing the pulling speed. The small deviation of the one from the theory at $c_{0}=$ $0.1 \%$ is due to the subcritical hysteresis [16].

By using this model, because the rather large interface thickness is used, the morphological transitions from a planner interface to shallow cells and further to deep cells can be easily simulated without the solute trapping problem. 


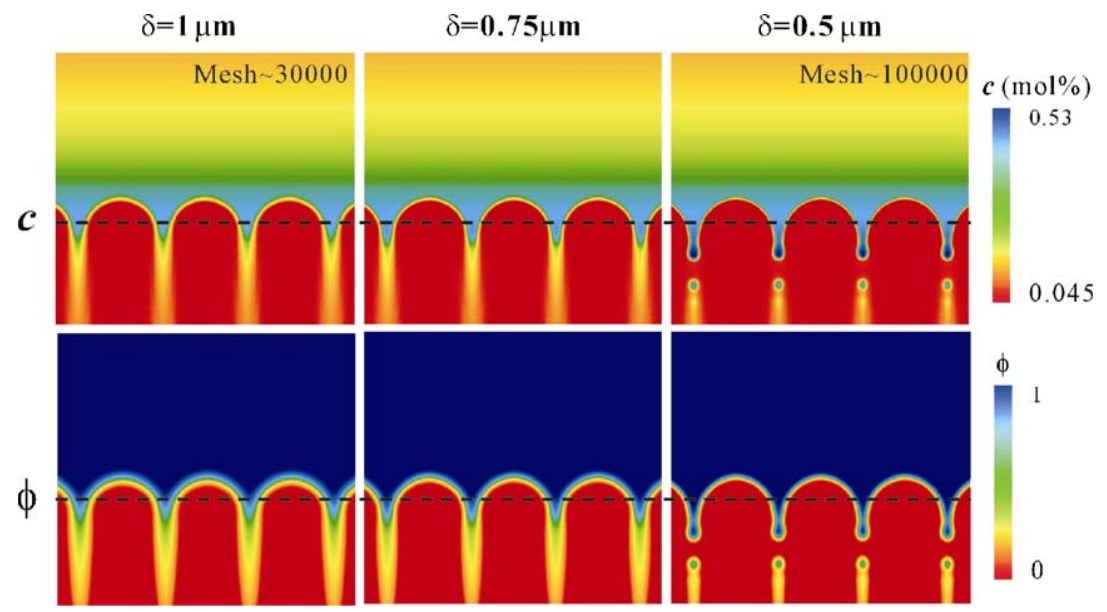

Fig. 7. The calculated phase field variable and concentration fields under different interface thicknesses.

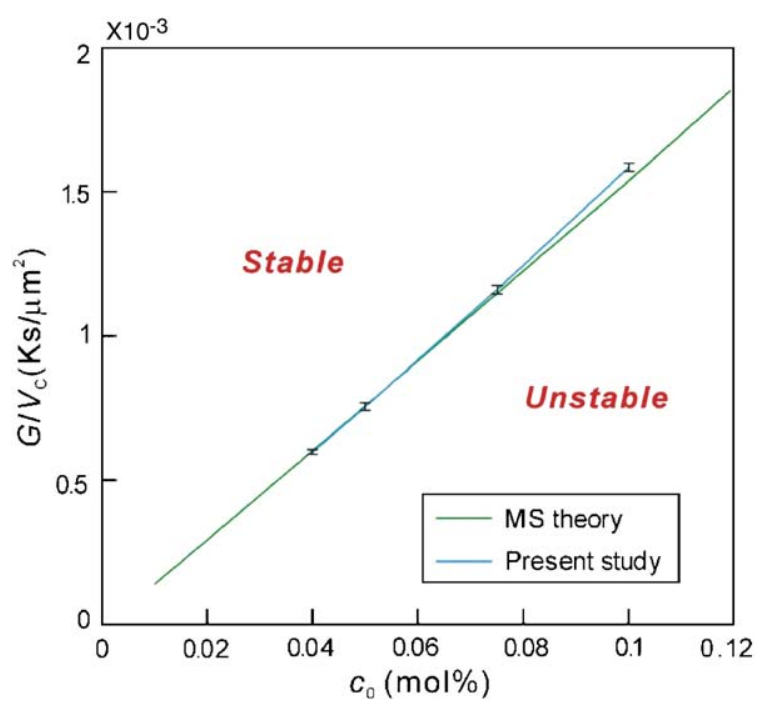

Fig. 8. Comparison of calculated critical thermal-gradient to speed ratio $(G / V)_{c}$ with the ones predicted by MS theory for different acetone concentrations.

Sample calculations $\left(c_{0}=0.05 \mathrm{~mol} \%\right.$ and $G=$ $100 \mathrm{~K} / \mathrm{cm}$ ) for the deep and shallow cells are shown in Fig. 9 for their concentration profiles. The simulated tip structure is almost independent of the interface thickness as long as the local curvature can be resolved. As the pulling speed is increased and a deep cellular interface emerges, the solute diffusion behavior is significantly changed.
As shown in Fig. 9, because of the narrow distance between the two tips and the thinner solutal boundary layer, concentration contours are very different from the ones in shallow cells. The solute ejected from side tips of deep cells makes the contours more concave, while the ones of shallow cells are linear. This is similar to the front-tracking results obtained by Ungar and Brown [17]. Besides, the simulated cell shapes under different pulling speeds are very similar to the ones observed experimentally [18]. Furthermore, for quantitative simulations, the agreement with the local G-T condition is important. To verify this, we have extracted local concentration distribution, tip radius $\rho$, and interface temperature $T_{i}$ of a single tip under various pulling speeds. These calculated data are summarized in Table 1, and the interface temperatures obtained by the G-T equation without considering the kinetic effect are also included for comparison. As shown, they are in good agreement, and the deviation is smaller than $0.08 \mathrm{~K}$. Furthermore, the values of $k_{\mathrm{eff}}\left(c_{\mathrm{S}} / c_{\mathrm{L}}\right)$ are also very close to the equilibrium one $(0.1)$, which indicates that the local thermodynamic consistence is well approximated. Although the agreement is good, the accuracy in the curvature term is still difficult to evaluate due to its small contribution. The curvature term could become important under a small thermal gradient, which poses another difficulty in the simulation. 

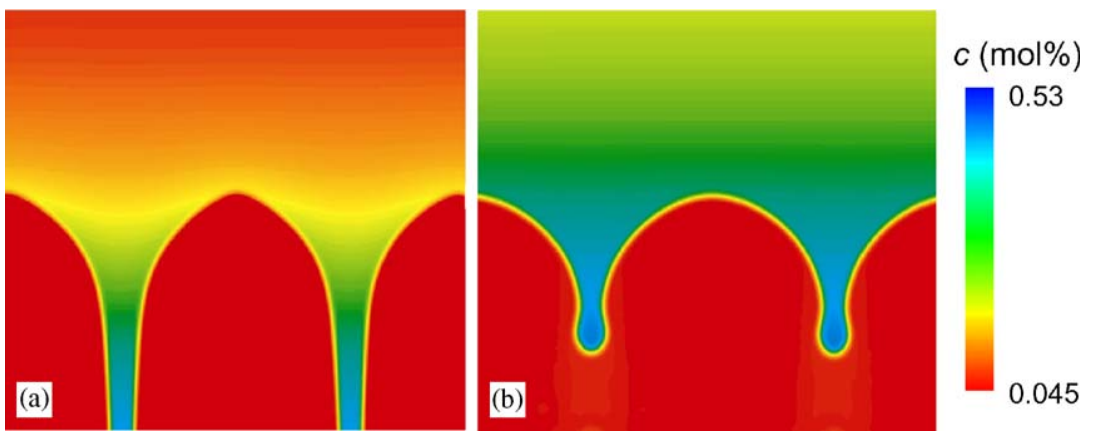

Fig. 9. Concentration distribution near the tips of (a) deep $\left(V=6.05 V_{c}\right)$ and (b) shallow cells $\left(V=1.36 V_{c}\right)$.

Table 1

Calculated tip radius, liquid and solid concentrations, and temperature for various pulling speeds. The tip temperatures derived from the Gibbs-Thompson (GT) equation are also summarized for comparison.

\begin{tabular}{llllll}
\hline$V(\mu \mathrm{m} / \mathrm{s})$ & $\rho(\mu \mathrm{m})$ & $c_{\mathrm{L}}(\mathrm{mol} \%)$ & $\mathrm{c}_{S}(\mathrm{~mol} \%)$ & $T_{i}$ & $\mathrm{~T}_{i,(\mathrm{G}-\mathrm{T} \text { equation) }}$ \\
\hline 27 & 7.692 & 0.2775 & 0.0280 & 330.576 & 330.634 \\
30 & 7.530 & 0.2594 & 0.0263 & 330.615 & 330.674 \\
35 & 7.837 & 0.2342 & 0.0237 & 330.668 & 330.729 \\
40 & 8.026 & 0.2160 & 0.0220 & 330.706 & 330.769 \\
50 & 7.987 & 0.1910 & 0.0194 & 330.757 & 330.825 \\
60 & 6.711 & 0.1750 & 0.0178 & 330.787 & 330.864 \\
80 & 5.123 & 0.1542 & 0.0154 & 330.847 & 330.915 \\
\hline
\end{tabular}

\section{Conclusion}

We have presented a simple interface model that can be embedded into a phase field model to eliminate solute trapping. This approach allows the simulation using a thick interface, but still smaller than the cell tip radius, over a wide range of solidification speeds. By using this model, a benchmark study with the ATC model is performed for an isothermal alloy dendritic growth, and both convergence tip radius and tip speed are in good agreement. Based on the comparison, it is quite promising for us to calculate the long time scale directional solidification problem near the onset of instability, while the present study shows good agreement with the classical Mullins-Sekerka theory [11]. In the future, because of the great release of the computational load by using this model, the coupled solution with thermal fields is also straightforward.

\section{Acknowledgements}

This research is sponsored by the National Science Council of Taiwan. The constructive comments from the reviewer are highly appreciated.

\section{References}

[1] W.J. Boettinger, J.A. Warren, C. Beckermann, A. Karma, Annu. Rev. Mater. Res. 32 (2002) 163.

[2] A.A. Wheeler, W.J. Boettinger, G.B. McFadden, Phys. Rev. A 45 (1992) 7424.

[3] A. Karma, W.J. Rappel, Phys. Rev. E 53 (1996) 3017; A. Karma, W.J. Rappel, Phys. Rev. E 57 (1998) 4323.

[4] G. Amberg, Phys. Rev. Lett. 91 (2003) 265505.

[5] N. Provatas, N. Goldenfeld, J. Dantzig, Phys. Rev. Lett. 80 (1998) 3308.

[6] C.W. Lan, C.M. Hsu, C.C. Liu, Y.C. Chang, Phys. Rev. E 65 (2002) 061601.

[7] A. Karma, Phys. Rev. Lett. 87 (2001) 115701.

[8] C.W. Lan, C.J. Shih, Phys. Rev. E 69 (2004) 031601. 
[9] J.C. Ramirez, C. Beckermann, A. Karma, H.-J. Diepers, Phys. Rev. E 69 (2004) 051607.

[10] C. Beckermann, H.-J. Diepers, I. Steinbach, A. Karma, X. Tong, J. Comput. Phys. 154 (1999) 468.

[11] W.W. Mullins, R.F. Sekerka, J. Appl. Phys. 35 (1964) 444.

[12] M.J. Aziz, T. Kaplan, Acta. Metall. 36 (1988) 2335.

[13] N.A. Ahmad, A.A. Wheeler, W.J. Boettinger, G.B. McFadden, Phys. Rev. E 58 (1998) 3436.
[14] I. Loginova, Ph.D. Thesis, Royal Institute of Technology, Sweden, 2003.

[15] J.A. Warren, J.S. Langer, Phys. Rev. E 47 (1993) 2702.

[16] D.J. Wollkind, L.A. Segel, Philos. Trans. R. Soc. London 268 (1970) 351.

[17] L.H. Ungar, R.A. Brown, Phys. Rev. B 31 (1985) 5391.

[18] P. Kurowski, C. Guthmann, S. De Cheveigne, Phys. Rev. A 42 (1990) 7368. 